\title{
The Potential of the Josephson Energy
}

\section{G. Blamire ${ }^{1}$}

Received: 7 July 2020 / Accepted: 18 July 2020 / Published online: 24 July 2020

(C) The Author(s) 2020

\begin{abstract}
The concept of the Josephson energy emerges directly from the original predictions of Brian Josephson. Although it can be used to explain the behaviour of Josephson devices, the Josephson energy has no present applications. In this paper, we show that in the context of magnetic Josephson junctions, the Josephson energy offers a potential mechanism for controlling magnetic memory devices.
\end{abstract}

Keywords Josephson junctions $\cdot$ Memory devices

\section{Introduction}

There can be comparatively few physicists (outside the USA at least) who were taught as undergraduates by two Nobel laureates. Cambridge in the early 1980s did offer this attraction-Anthony Hewish lectured the entire physical science class at the start of the first year on special relatively but of course told us about the discovery of pulsars for which he won his prize. This was presumably a deliberate ploy to reinforce the idea that physics was exciting - and perhaps it succeeded in that I was originally intending to study chemistry. In contrast, lectures from Brian Josephson were reserved for an optional final year course on superconductivity. This was taken only by quite a small number of students; while this may have been partly because of Brian's much more unassuming style, I think it reflected more the prevailing sense of the time that superconductivity had been "finished." Given the types of experiment which could be performed during that period this was perhaps largely true, the BCS theory and Brian's own work $[1,2]$ provided a more or less complete understanding of the underlying phenomena; more technical issues such as improving the properties of superconducting wire through improved flux pinning were also understood at a fundamental level [3], and so improving practical superconductors for applications was something which could safely be left to materials scientists and engineers.

M. G. Blamire

mb52@cam.ac.uk

1 Department of Materials Science, University of Cambridge, 27 Charles Babbage Road, Cambridge CB3 0FS, UK
The idea of superconductivity being a dead subject was radically altered in 1986 by the discovery of hightemperature superconductivity (HTS) [4]. Although it was clear immediately that the underlying mechanism of HTS was substantially different to BCS, experiments on junctions (mainly artificial grain boundary devices [5]) showed that the Josephson equations remained valid and so enabled the development of practical devices such as HTS SQUIDS [6].

In parallel with the discovery of new classes of HTS materials, the development of improved thin film deposition techniques was enabling the growth of complex device heterostructures. Driven by a technological need to replace lead with niobium in the superconducting electronics then under development, this work led to the creation of the first artificial tunnel barriers [7] and complex multi-barrier [8] and multilayer devices [9]. This work ultimately enabled the creation of Josephson devices in which one or more constituent layers were ferromagnetic [10-13]. Such ferromagnetic Josephson junctions (FJJs) have a number of distinct properties which can give rise to radically different properties and interesting potential applications [14]. This article discusses FJJs in the context of the original predictions by Brian Josephson and ends with a discussion of the potential application of the Josephson energy to FJJs.

\section{Magnetic Barriers}

The most definitive test of the dc Josephson effect is the modulation of the critical current by an applied magnetic field. For a junction with a typical length scale of a few micrometres, the application of fields of a few $\mathrm{mT}$ in a direction normal to the 
current flow leads to a substantial oscillation of the supercurrent magnitude which can be used to distinguish uniform Josephson coupling from the presence, for example, of localized shorts in a tunnel barrier. This behaviour results from the flux induced by the field generating a spatial gradient in the phase difference across the junction and for the standard example of a rectangular junction leads to the well-known Fraunhofer oscillation of $I_{c}(H)$ [15].

A ferromagnetic barrier has an internal flux density associated with the magnetization which will add vectorially to that induced by an external applied field. In the simplest case of a saturated magnetic barrier aligned either parallel or antiparallel to the applied field, the total barrier flux will be greater or less respectively than that provided by the field on its own. This results in a hysteretic shift in the Fraunhofer pattern triggered at the coercive field of the barrier [16].

Such behaviour has been widely observed in FJJs with barriers with a square ferromagnetic hysteresis loop [17]. More complicated behaviour occurs for barriers with a more gradual ferromagnetic reversal or ones entering a multidomain state [16].

\section{Pi-Junctions}

The singlet superconducting state in which the electron Cooper pairs comprise antiparallel spins is strongly suppressed by the exchange field associated with ferromagnetism which favours parallel spin alignment. Consequently, proximity-induced superconductivity in a ferromagnet is strongly depressed compared with the equivalent superconductor/normal metal interface, and the interfacial superconductivity on the $\mathrm{S}$ side is significantly weakened. For superconductor/metallic ferromagnetic interfaces, complex effects described by the Larkin-Ovchinnikov-Ferrel-Fulde (LOFF) $[18,19]$ theory are present.

The most direct demonstration of these LOFF effects occurs in superconductor/ferromagnet/superconductor FJJs. The wave function interference effect leads to an oscillation with ferromagnet thickness in the ground state phase difference of the order parameter across the junction. In such junctions, the critical current oscillates as it decays with ferromagnet thickness [17]. Such measurements measure only the magnitude of the critical current and so the periodic nodes in the plot represent transitions between positive and negative critical currents ( 0 - and $\pi$-states). The ability to generate a ground state $\pi$ state by appropriately selecting the ferromagnetic barrier thickness (as opposed to inducing one by the application of magnetic field) is of potential benefit in quantum computing applications $[20,21]$.

The effective ferromagnet thickness may be altered in a single device by creating a $\mathrm{S} / \mathrm{F} / \mathrm{N} / \mathrm{F} / \mathrm{S}$ spin valve Josephson junction (SVJJ) [22]. Here, the total ferromagnet thickness adds when the magnetization of the F layers is parallel and subtracts in the antiparallel state. This potentially provides a memory function in which the magnetic state controls the ground state phase difference of the device. The first experiments on SVJJs demonstrated an ability to switch the critical current magnitude by altering the magnetic state (and hence the net flux within the junction) via the application of a magnetic field [22]. This has been explored as a technological basis for a cryogenic magnetic memory, but the ability to modify the ground state phase difference rather than the magnitude of the critical current offers a more direct integration with conventional and quantum superconducting electronics [23]. However, it is known from work on magnetoresistive memory that the field-driven magnetic switching presents issues of accurately addressing individual memory cells [24] and is not size scalable.

\section{Spin Valve Josephson Junctions and the Josephson Energy}

The Josephson coupling energy $\left(E_{J}\right)$ can be derived [25] from the original Josephson equations $[1,2]$.

$I_{S}=I_{c} \sin (\Delta \phi)$

and

$d(\Delta \phi) / \mathrm{dt}=2 e V / \hbar$

(where $\Delta \phi$ is the phase difference across the junction, $I_{S}$ and $I_{c}$ are the superconducting pair current and the critical current respectively, and $V$ is voltage across the junction and $\hbar$ is the Planck constant) to give the free energy stored in the junction from electrical work

$$
\begin{aligned}
F & =\int I_{S} V d t=\left(I_{c} \hbar / 2 e\right) \int \sin (\Delta \phi) d(\Delta \phi) \\
& =\text { const. }-E_{J} \cos (\Delta \phi)
\end{aligned}
$$

where $E_{J} \equiv I_{C} \hbar / 2 e$. Substituting for $\Delta \phi$ from (1) gives:

$F=$ const $-\frac{\hbar}{2 e} \sqrt{I_{c}{ }^{2}-I_{S}{ }^{2}}$

This equation is conventionally used to determine the onset of junction phase-slip - either by $I_{S}$ exceeding the critical current $I_{c}$ or when thermal activation $k_{B} T \sim I_{c}-I_{S}$ where $k_{B}$ is the Boltzmann constant and $T$ is the temperature.

Here, we explore the potential for the Josephson energy to be used directly to control the magnetic state of a device with possible potential applications. We start by generalizing (4), making $I_{c}$ dependent on the magnetic state-for example, for a SVJJ, the critical current for the antiparallel configuration $\left(I_{C A P}\right)$ would generally be higher than the parallel state 
configuration $\left(I_{c P}\right)$. At a current $I_{S} \sim I_{c P}$, the 2 nd term in (4) would approach zero whereas at the same current $I_{S}<I_{c A P}$, and so the 2 nd term would be finite. The free energy difference

$\Delta F=\frac{\hbar}{2 e}\left(\sqrt{I_{c P}^{2}-I_{S}^{2}}-\sqrt{I_{C A P^{2}-I_{S}^{2}}}\right)$

therefore provides an effective exchange coupling between the magnetic layers which acts to drive them towards an AP state.

In action, this is analogous to the superconducting exchange coupling (SEC) predicted [26] and realized [27] in ferromagnetic insulator/superconductor/ferromagnetic insulator $(\mathrm{FI} / \mathrm{S} / \mathrm{FI})$ spin valves in which changes in the superconducting condensation energy of the middle layer, due the addition or cancellation of the magnetic exchange coupling with the FI layers, tend to favour AP alignment of the FI layers.

As a potential memory device, this clearly leaves some things to be desired. In this form, writing is enabled with either current direction and can only drive the device in one direction (i.e., $\mathrm{P} \rightarrow \mathrm{AP}$ ). The device could be non-destructively read in the AP state (i.e., a measurement of $I_{c}$ would yield $I_{c A P}$ ) but not in the $P$ state because the switching current must be smaller than $I_{c P}$. As a test of the principle of using the Josephson energy to drive magnetic switching, the latter concern could be overcome by performing read measurements at a higher temperature which would reduce both the critical currents and the free energy available for driving the magnetic switching.

It is likely to be challenging even to demonstrate this level of functionality. In terms of driving magnetic switching for a particular $\mathrm{F}$ layer thickness, the crucial parameter is the change in free energy by unit area, $\Delta F / A$, and so this requires the highest possible critical current density $J_{c}$. FJJs tend to have low values of $J_{c}$, but if we take a reasonably large value amongst those reported in the literature (for single barrier $\mathrm{S} / \mathrm{F} /$ S FJJs) of $10^{9} \mathrm{~A} \mathrm{~m}^{-2}$, we would expect a maximum value of $\Delta F / A$ to be of the order of $3 \times 10^{-7} \mathrm{~J} \mathrm{~m}^{-2}$. This is two orders of magnitude lower than the free energy change associated with SEC [27], and so either a magnetic material with very low switching energy is required, or much higher critical current densities will be needed.

\section{A Toy Model for a Josephson Magnetic Memory}

Assuming that appropriate magnetic materials could be developed, it is possible to design a device structure which could enable bidirectional writing with the potential for nondestructive readout. One example, albeit with obvious issues regarding scalability, is set out here.
From the point of view of bidirectional writing, the problem with Eq. (4) is the lack of sensitivity to current direction. This could be overcome if $I_{c}$ became an odd function of current. One example of this, the so-called long Josephson junction (LJJ), is well known. In the LJJ, the junction is large enough that the flux within the junction generated by the junction bias current is of a similar order to the flux quantum [15]. Under these circumstances, the Fraunhofer $I_{c}(H)$ pattern for a conventional $\mathrm{JJ}$ is asymmetrically distorted because the current-induced flux adds to, or subtracts from, that due to the applied field depending on the current direction. Therefore, a LJJ in an appropriate external bias field $H$ will have a critical current which is current direction dependent.

Assuming for simplicity that $I_{c}$ is linear with $\mathrm{H}$ in the operating region of the device, we can extend Eq. (4) for a ferromagnetic LJJ as

$$
F=\text { const }^{-} \frac{\hbar}{2 e} \sqrt{\left(I_{c 0} \pm \alpha M I_{S}\right)^{2}-I_{S}^{2}}
$$

where $I_{c 0}$ is the zero-field critical current, $M$ is the barrier magnetization, and $\alpha$ is a coupling constant proportional the Oersted field induced by the bias current and the barrier magnetization $(M)$ coupling to the junction. $F$ is sketched in Fig. 1 for the two directions of barrier magnetization.

As indicated in the figure, the system can gain free energy by switching between $\pm \mathrm{M}$ states provided that the energy required for magnetic switching $\Delta F_{\text {switch }}$ is sufficiently small. As can be seen from the diagram, switching is directional with positive and negative currents switching to negative and positive magnetic states, respectively. Note that for simplicity, this model ignores the effect of the Oersted field on the barrier magnetization.

Reading such a device would still present a challenge because the switching current (in one direction at least) is lower

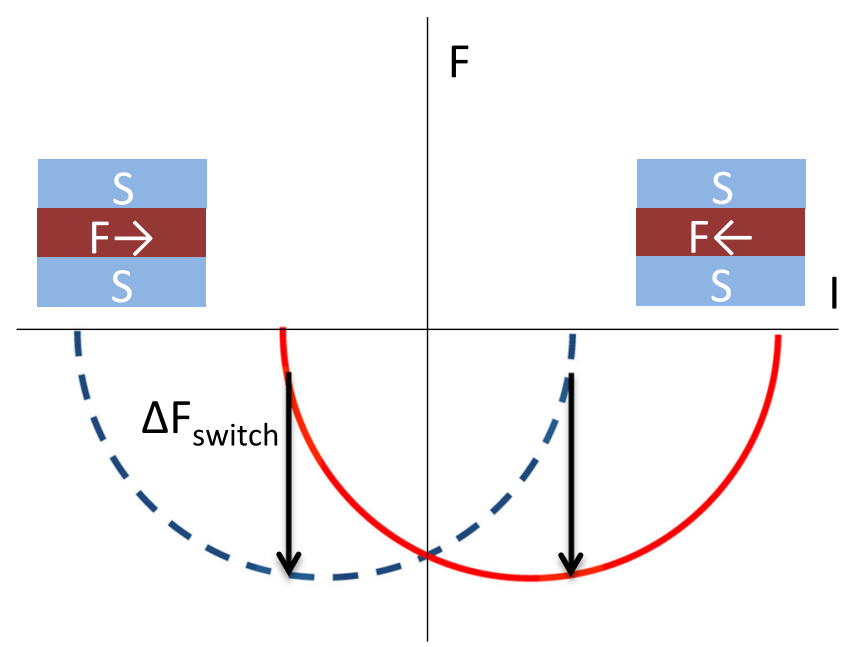

Fig. 1 A sketch of free energy versus bias current for a ferromagnetic long Josephson junction for the two barrier magnetization directions. $\Delta F_{\text {switch }}$ is the switching energy for the barrier magnetization 
than the critical current. This could be overcome if read current pulses were used which were shorter than the characteristic magnetic reversal time.

Acknowledgements This work was supported by EPSRC Programme Grant No. EP/N017242/1.

Open Access This article is licensed under a Creative Commons Attribution 4.0 International License, which permits use, sharing, adaptation, distribution and reproduction in any medium or format, as long as you give appropriate credit to the original author(s) and the source, provide a link to the Creative Commons licence, and indicate if changes were made. The images or other third party material in this article are included in the article's Creative Commons licence, unless indicated otherwise in a credit line to the material. If material is not included in the article's Creative Commons licence and your intended use is not permitted by statutory regulation or exceeds the permitted use, you will need to obtain permission directly from the copyright holder. To view a copy of this licence, visit http://creativecommons.org/licenses/by/4.0/.

\section{References}

1. Josephson, B.D.: Phys. Lett. 1, 251 (1962)

2. Josephson, B.D.: Adv. Phys. 14, 419 (1965)

3. Campbell, A.M., Evetts, J.E.: Adv. Phys. 21, 199 (1972)

4. Bednorz, J.G., Muller, K.A.: Z. Phys. B. 64, 189 (1986)

5. Chaudhari, P., Mannhart, J., Dimos, D., Tsuei, C.C., Chi, J., Oprysko, M.M., Scheuermann, M.: Phys. Rev. Lett. 60, 1653 (1988)

6. Laibowitz, R.B., et al.: Appl. Phys. Lett. 56, 686 (1990)

7. Gurvitch, M., Washington, M.A., Huggins, H.A.: Appl. Phys. Lett. 42, 472 (1983)
8. Blamire, M.G., Somekh, R.E., Morris, G.W., Evetts, J.E.: IEEE Trans. Magn. 25, 1135 (1989)

9. Goldie, D.J., Booth, N.E., Patel, C., Salmon, G.L.: Phys. Rev. Lett. 64, 954 (1990)

10. Ryazanov, V.V., Oboznov, V.A., Rusanov, A.Y., Veretennikov, A.V., Golubov, A.A., Aarts, J.: Phys. Rev. Lett. 86, 2427 (2001)

11. Robinson, J.W.A., Witt, J.D.S., Blamire, M.G.: Science. 329, 59 (2010)

12. Keizer, R.S., Goennenwein, S.T.B., Klapwijk, T.M., Miao, G., Xiao, G., Gupta, A.: Nature. 439, 825 (2006)

13. Bell, C., Loloee, R., Burnell, G., Blamire, M.G.: Phys. Rev. B. 71(R), 180501 (2005)

14. Buzdin, A.I.: Rev. Mod. Phys. 77, 935 (2005)

15. Barone, A., Paterno, G.: Physics and Applications of the Josephson Effect. Hoboken, Wiley (1982)

16. Blamire, M.G., Smiet, C.B., Banerjee, N., Robinson, J.W.A.: Supercond. Sci. Technol. 26, 055017 (2013)

17. Robinson, J.W.A., Piano, S., Burnell, G., Bell, C., Blamire, M.G.: Phys. Rev. Lett. 97, 177003 (2006)

18. Larkin, A., Ovchinnikov, A.: Sov. Phys. JETP. 20, 762 (1965)

19. Fulde, P., Ferrell, R.A.: Phys. Rev. 135, A550 (1964)

20. Feofanov, A.K., et al.: Nat. Photonics. 6, 593 (2010)

21. Frolov, S.M., et al.: Nat. Phys. 4, 32 (2007)

22. Bell, C., Burnell, G., Leung, C.W., Tarte, E.J., Kang, D.J., Blamire, M.G.: Appl. Phys. Lett. 84, 1153 (2004)

23. Madden, A.E., Willard, J.C., Loloee, R., Birge, N.O.: Supercond. Sci. Technol. 32, 015001 (2019)

24. Engel, B.N., et al.: IEEE Trans. Magn. 41, 132 (2005)

25. Tinkham, M.: Introduction to Superconductivity. McGraw-Hill, New York (1996)

26. de Gennes, P.G.: Phys. Lett. 23, 10 (1966)

27. Zhu, Y., Pal, A., Blamire, M.G., Barber, Z.H.: Nat. Mater. 16, 195 (2017)

Publisher's note Springer Nature remains neutral with regard to jurisdictional claims in published maps and institutional affiliations. 